\title{
Retinal vessel segmentation using a probabilistic tracking method
}

\author{
Yi Yin, Mouloud Adel and Salah Bourennane \\ Institut Fresnel, UMR-CNRS 6133, Ecole Centrale Marseille, Université Paul Cézanne \\ Domaine Universitaire de Saint-Jérôme, 13397, Marseille Cedex 20, France
}

\begin{abstract}
Vessel structures such as retinal vasculature are important features for computeraided diagnosis. In this paper, a probabilistic tracking method is proposed to detect blood vessels in retinal images. During the tracking process, vessel edge points are detected iteratively using local grey level statistics and vessel's continuity properties. At a given step, a statistic sampling scheme is adopted to select a number of vessel edge points candidates in a local studying area. Local vessel's sectional intensity profiles are estimated by a Gaussian shaped curve. A Bayesian method with the Maximum a posteriori (MAP) probability criterion is then used to identify local vessel's structure and find out the edge points from these candidates. Evaluation is performed on both simulated vascular and real retinal images. Different geometric shapes and noise levels are used for computer simulated images, whereas real retinal images from the REVIEW database are tested. Evaluation performance is done using the Segmentation Matching Factor (SMF) as a quality parameter. Our approach performed better when comparing it with Sun's and Chaudhuri's methods. ROC curves are also plotted, showing effective detection of retinal blood vessels (true positive rate) with less false detection (false positive rate) than Sun's method.
\end{abstract}

Keywords: retinal image, edge detection, vessel tracking, Bayesian segmentation

\section{Introduction}

The automatic analysis of blood vessels is a very important task in many clinical investigations and scientific research related to vascular features. The 
early diagnosis of several pathologies, such as arterial hypertension, arteriosclerosis or diabetic retinopathy could be achieved by analyzing the vascular structures. Moreover for many clinical investigations, vessel segmentation is becoming a prerequisite for the analysis of vessel parameters such as tortuosity and variation of the vessel width along the vessel and the ratio between the venous and arterial vessel width. Many vessel extraction methods, using different approaches, have been reported in the literature. A major review of these methods can be found in [1]. The techniques published in the research literature in response to the importance of retinal vessel extraction may be roughly categorized into methods based on matched filters [2-8], adaptive thresholds [3, 9], intensity edges [10, 11], region growing [12], statistical inferencing [13], mathematical morphology [12, 14, 15], and Hessian measures [16-19].

Most of the work on vessel segmentation techniques can be divided into two main groups : Pixel-based methods and tracking methods. An advantage of tracking based methods is the guaranteed connectedness of vessel segments whereas in pixel processing based methods, connectedness is not guaranteed. A number of features are computed for every pixel in an image in the case of pixel processing based methods. Based on these features, each pixel is assigned a label that indicates the probability that the pixel is inside a vessel. Various supervised [18, 20, 21] and unsupervised [22-25] pixel labeling frameworks have been proposed. Since each pixel is classified independently, a disadvantage of such methods is that gaps can appear in the segmented vessels resulting in an unconnected vessel network [26].

Vessel trackers choose seed points on the edges and the centrelines of vessels, either automatically or manually, and follow vessels from these points $[4,10,27,28]$. Given the location and direction of a vessel, they take a small step in the direction of the vessel and look for the vessel edges or the centerlines or both nearby [29]. When new edges are found, an estimate of vessel direction is made, and a new step is made in this direction. As vessels are connected in the retina, tracking methods can follow a whole tree without examining the vast majority of the image that does not contain vessel. Vessel tracking can thus give information on vessel structure such as branching and connectivity. Vessel trackers must however include some logic to respond to vessel endpoints and to branch points.

Techniques used for identifying vessels are based on matched filters [10], morphological filters [15], optimization of Gaussian profiles [30] or fuzzy cmean classifiers [31], Bayesian inference [24, 27]. Other techniques combining 
the two approaches described above have been also used to improve performance [20, 22, 23]. Among tracking methods, few probabilistic approaches have been reported in the literature of vessel segmentation [32]. The main ideas of a probabilistic tracking method have been presented in our previous article [33], which needed some improvements like modeling the blood vessel more accurately and handling different vessel configurations.

Two stages are needed for a tracking method to detect the whole vascular tree. The first one is the selection of seed points all over the retinal image. The second one is based on tracking from these seed points and combining the results of local vessel segmentation to get the final detected vascular tree. Local vessel segmentation [34-36] is the foundation to segment the whole vascular tree. Our study focuses on this second stage, which takes into account local vessel detection, including the vessel edges and centerline detection. In this paper, a novel improved tracking-based method using a probabilistic formulation is introduced. The new approach uses a Gaussian model to approximate the vessel's sectional intensity profile, identifies bifurcation and crossing configurations, and improves the detection results as shown in the experiments and discussion section. A probabilistic segmentation scheme is associated with the Maximum a posteriori (MAP) as criterion to estimate local vessel edges. In the following, section II gives a general description of our method and explains the concept of statistical sampling model. Bayesian segmentation is described in section III. Finally results and discussion are given in section IV.

\section{Method Description}

\subsection{General description}

The proposed method is based on an iterative tracking algorithm. The tracking process starts from an initial point which is selected manually by the user. Vessel edge points are then detected iteratively using local grey levels statistics and the information obtained from the previous iterations. This process stops when current blood vessel ends or the vessel branches are found. All found branches are considered as new blood vessels and are processed by the same iterative algorithm. Many methods have been used to find branching in a vascular tree and endpoints when tracking blood vessels $[10,27,37]$. The logic to detect these important features is based on center line extraction [10], filter response [27], or diameter computation [37] for branching and branch detection [27] or poor contrast [37] for endpoint 
localization. None of them uses probabilistic consideration as the case in our approach.

For clarity, the tracking process is described as follows:

- Initialization: In practice, two initial edge points are chosen manually diametrically opposed on the interested blood vessel. Center point is chosen and initial tracking direction is set along local vessel's direction.

- Iteration: At current iteration, local blood vessel parameters including vessel edge points, center point, direction and diameter are supposed to be obtained in the previous iteration. A search window with a statistical sampling method is then used to select the most probable vessel configuration (normal, bifurcation or crossing) using Bayesian theory with MAP as a criterion. This selection is done when testing all the possible configurations with 2 edge points (normal), 4 edge points (bifurcation), or 6 edge points (crossing). Blood vessel parameters including edge points, direction, and diameter are then obtained for the next iteration. The configuration models will be described in detail below.

- End: When the blood vessel diameter is less than one pixel, the current vessel's end is found and the tracking of the current vessel stops. Besides, when the vessel branches are found, all initial information of the branches are obtained by the algorithm. In this situation, the current tracking process stops, and the tracking of these branches, which are considered as new blood vessels, starts.

\subsection{Dynamic Search Window}

At a given step, a semi-ellipse drawn in the current studying area is regarded as a search window (see Fig.1). In order to deal with the complex geometrical features of the retinal blood vessels, the semi-ellipse should be dynamic and self-adapting during the tracking process. When vessel's diameter increases, the semi-ellipse should be enlarged in order to cover the potential position of the new edge points. When vessel's diameter decreases, we should reduce it to avoid inaccurate detection, such as detecting another vessel near the current one. Besides, the minor axis of the ellipse, which is parallel to local vessel's direction, could denote the look-ahead distance. To make the algorithm robust to highly curved blood vessel, the minor axis is set adaptive to local vessel's curvature. A smaller minor axis is chosen when 
vessel's curvature increases, so that the tracking direction could catch up with vessel's change more easily.

At iteration $k$, vessel edge points $\hat{U}_{k}, \hat{V}_{k}$, center point $O_{k}$, direction $\overrightarrow{D_{k}}$ and diameter $d_{k}$ are known parameters. $O_{k}$ is the middle point of $\left[\hat{U}_{k}, \hat{V}_{k}\right]$. $\overrightarrow{D_{k}}$ heads towards $\overrightarrow{O_{k-1} O_{k}}$ and is defined as a unit vector. Local diameter is $d_{k}=\left|\hat{U}_{k} \hat{V}_{k}\right|$. A semi-ellipse $C_{k}$ is defined to be centered on $O_{k}$ and heading towards $\overrightarrow{D_{k}}$. As shown in Fig.1, its major axis $a_{k}$ is perpendicular to $\overrightarrow{D_{k}}$ while the minor axis $b_{k}$ is parallel to it. $a_{k}$ and $b_{k}$ are fixed as follows:

$$
\left\{\begin{array}{l}
a_{k}=\alpha d_{k} \\
b_{k}=\beta\left(\pi-\theta_{k}\right) d_{k}
\end{array}\right.
$$

where $\theta_{k}$ is the angle between the current and previous vessel directions: $\theta_{k}=\arccos \left(\overrightarrow{D_{k-1}} \cdot \overrightarrow{D_{k}}\right) \cdot \theta_{k}$ provides the information of local vessel's curvature. $\alpha, \beta$ are constant factors whose values have been set based on the simulated results. In this study, $\alpha$ was fixed to 2 , and $\beta$ was set to $1 / \pi$ so that the magnitude of the minor axis is less than local vessel's diameter.

\subsection{Configuration Model}

A number of edge points candidates are selected on the semi-ellipse search window. In order to choose new edge points among these candidates, we define configuration models which combine the points candidates with vessel's structures. In this study, we categorize vessel's structures into three types of configurations: normal, bifurcation and crossing. The normal case is regarded as the situation in which only a single vessel exists in current search region. The case of bifurcation means that one single vessel is divided into two branches. A crossing case is described when one vessel overlaps another.

At iteration $k$, we select $N_{k}$ points which are numbered from 1 to $N_{k}$ on the semi-ellipse $C_{k}$ (see Fig.2). A configuration $\chi$ is defined by a set of edge points candidates. Two points selected from the $N_{k}$ ones are called a normal configuration $\chi_{n}$. A normal configuration as shown in Fig.2(a) has two selected points $M_{m_{1}}$ and $M_{m_{2}}$, which are the $m_{1}^{t h}$ and $m_{2}^{\text {th }}$ points on $C_{k}$ $\left(1<m_{1}<m_{2}<N_{k}\right)$. They are assumed as two edge points and divide the $N_{k}$ points on $C_{k}$ into three point sets. So, $M_{i}$, the $i^{t h}$ point on $C_{k}\left(i \in\left[1, N_{k}\right]\right)$, is assumed to belong to the blood vessel if $i \in\left[m_{1}, m_{2}\right]$ or to the background if $i \in\left[1, m_{1}\right) \cup\left(m_{2}, N_{k}\right]$. For a bifurcation configuration $\chi_{b}$, four points are selected on $C_{k}$ to describe the edge points of two branches. Six points are 
needed for a crossing configuration $\chi_{c}$. Two of them are considered as the new edge points of the same vessel, while the other four points are considered as the edge points of another vessel which is over or under the current one.

There are $C_{N_{k}}^{2}=\frac{N_{k} \text { ! }}{2 !\left(N_{k}-2\right) !}$ normal configurations at iteration $k$. It is the number of 2-combinations from the set of $N_{k}$ points candidates. The numbers of the bifurcation and crossing configurations are $C_{N_{k}}^{4}$ and $C_{N_{k}}^{6}$ respectively. Thus, the total number of configurations $S_{k}$, at iteration $k$ is:

$$
S_{k}=C_{N_{k}}^{2}+C_{N_{k}}^{4}+C_{N_{k}}^{6}
$$

\section{Bayesian Method for Vessel Segmentation}

During the tracking process, vessel edge points are obtained by comparing the probabilities of different configurations. When the configuration with the maximum probability is obtained, selected points used to define it are considered as vessel edge points. At iteration $k$, the probability of a configuration $\chi$ is described by $P\left(\chi \mid Y_{k}\right)$, where $Y_{k}=\left\{y_{s}, s=1,2 \ldots N_{k}\right\}$ is the discrete grey level profile corresponding to the $N_{k}$ points on semi-ellipse $C_{k}$. Based on the Maximum a posteriori (MAP) criterion, the best configuration $\hat{\chi}$ is the one which has the maximum probability among all the $S_{k}$ ones (see Eq.2), $\hat{\chi}=\arg \max \left\{P\left(\chi \mid Y_{k}\right)\right\}$. With Bayes' rule:

$$
P\left(\chi \mid Y_{k}\right)=\frac{P\left(Y_{k} \mid \chi\right) \cdot P(\chi)}{P\left(Y_{k}\right)}
$$

When $N_{k}$ points are selected, $Y_{k}$ has a fixed value, so $P\left(Y_{k}\right)$ does not depend on the configuration and will be disregarded. The best configuration is:

$$
\hat{\chi}=\underset{\chi}{\arg \max }\left\{P\left(Y_{k} \mid \chi\right) \cdot P(\chi)\right\}
$$

If $\hat{\chi}$ is a normal configuration, the two points candidates used to define $\hat{\chi}$ are regarded as new edge points $\hat{U}_{k+1}, \hat{V}_{k+1}$ respectively. If $\hat{\chi}$ is a bifurcation configuration, four corresponding points are considered as the initial edge points of two branches. If $\hat{\chi}$ is a crossing configuration, the third and fourth points of the six ones corresponding to $\hat{\chi}$ are regarded as new edge points $\hat{U}_{k+1}, \hat{V}_{k+1}$ of current blood vessel. The other four points are considered as the initial edge points of these two branches starting from the crossing. 


\subsection{Likelihood Function}

$P\left(Y_{k} \mid \chi\right)$ is the conditional probability of the discrete grey levels profile $Y_{k}$ for a given configuration $\chi$. Assuming that the discrete grey levels on the semi-ellipse are independent,

$$
P\left(Y_{k} \mid \chi\right)=\prod_{i=1}^{N_{k}} P\left(y_{i} \mid \chi\right)
$$

We suppose the conditional probability model $P\left(y_{i} \mid \chi\right)$ describes the variability of the $i^{\text {th }}$ point on $C_{k}$ belonging either to the background or to the blood vessel.

We consider first the case of a normal configuration $\chi_{n}$ as shown in Fig.2(a). $M_{m_{1}}$ and $M_{m_{2}}$ are the $m_{1}^{\text {th }}$ and $m_{2}^{\text {th }}$ points selected on semi-ellipse $C_{k}$. Likelihood function is expressed as:

$$
P\left(Y_{k} \mid \chi_{n}\right)=\prod_{i=1}^{m_{1}-1} P\left(y_{i} \mid b\right) \prod_{m_{1}}^{m_{2}} P\left(y_{i} \mid v\right) \prod_{m_{2}+1}^{N_{k}} P\left(y_{i} \mid b\right)
$$

where $b$ and $v$ denote the background and vessel respectively and will be used in the following sections. In our method, local background is assumed to have a constant intensity. In retinal image, vessel's sectional intensity profile can be approximated as a Gaussian shaped curve [2], [38]. Thus, for a points $M$ in current search area, grey level $x_{M}$ is expressed as:

$$
x_{M}= \begin{cases}G(M)=(A-B) \exp \left(-\frac{l^{2}}{2 \sigma^{2}}\right)+B & \text { if } \quad M \in v \\ B & \text { if } \quad M \in b\end{cases}
$$

As shown in Fig.3(a), $A$ is the grey level of local vessel's center, $B$ is the grey level of local background, $l$ is the perpendicular distance between $M$ and the straight line which passes through vessel's center along local vessel direction, and $\sigma$ defines the spread of the sectional intensity profile and is set to half of local vessel's radius. Fig.3(b) shows the estimated intensity profile of a given vessel cross-section, which is obtained by the proposed Gaussian intensity model.

For the normal configuration $\chi_{n}$ mentioned above, estimated grey level 
of $M_{i}$, the $i^{t h}$ point on $C_{k}$, is described as:

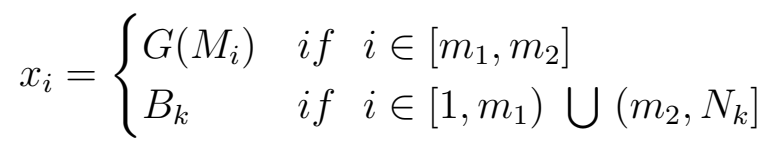

When $i \in\left[m_{1}, m_{2}\right], M_{i}$ is assumed to belong to the blood vessel. Its grey level is estimated by the proposed Gaussian intensity model: $G\left(M_{i}\right)=\left(A_{k}-\right.$ $\left.B_{k}\right) \exp \left(-l_{i}^{2} /\left(2 \sigma_{\chi_{n}}^{2}\right)\right)+B_{k} . A_{k}$ and $B_{k}$ are the local intensity parameters at iteration $k$. When $k \geq 5, A_{k}$ and $B_{k}$ are calculated on three moving regions: one inside and two others outside the local blood vessel. As shown in Fig.4, three regions are restricted by five pairs of edge points obtained in previous iterations. $A_{k}$ is the mean grey level of the region surrounding local vessel center line. $B_{k}$ is the mean grey level of two regions in local background. When $k<5, A_{k}$ and $B_{k}$ are obtained by three regions chosen manually in initialization. $l_{i}$ is the distance between $M_{i}$ and the straight line which passes through the middle point of $\left[M_{m_{1}}, M_{m_{2}}\right]$ and is perpendicular to $\overrightarrow{M_{m_{1}} M_{m_{2}}}$. Spread parameter $\sigma_{\chi_{n}}=\left|M_{m_{1}} M_{m_{2}}\right| / 4$.

If we assume the retinal image affected only by additive white Gaussian noise $\xi$, the observed grey level of $M_{i}$ is supposed:

$$
y_{i}=x_{i}+\xi= \begin{cases}G\left(M_{i}\right)+\xi_{v} & \text { if } i \in\left[m_{1}, m_{2}\right] \\ B_{k}+\xi_{b} & \text { if } i \in\left[1, m_{1}\right) \bigcup\left(m_{2}, N_{k}\right]\end{cases}
$$

where $\xi_{v} \sim N\left(0, \sigma_{v}^{2}\right)$ and $\xi_{b} \sim N\left(0, \sigma_{b}^{2}\right)$ are the Gaussian noise in local blood vessel and background respectively. Standard deviation $\sigma_{v}$ and $\sigma_{b}$ are also obtained on three regions as shown in Fig.4. So conditional probability model of the given normal configuration is:

$$
\left\{\begin{array}{l}
P\left(y_{i} \mid v\right)=\frac{1}{\sqrt{2 \pi} \sigma_{v}} \exp \left(-\frac{\left(y_{i}-G\left(M_{i}\right)\right)^{2}}{2 \sigma_{v}^{2}}\right) \\
P\left(y_{i} \mid b\right)=\frac{1}{\sqrt{2 \pi} \sigma_{b}} \exp \left(-\frac{\left(y_{i}-B_{k}\right)^{2}}{2 \sigma_{b}^{2}}\right)
\end{array}\right.
$$

And the likelihood function $P\left(Y_{k} \mid \chi_{n}\right)$ is obtained by Eq.6.

For the case of bifurcation or crossing, the likelihood function is computed similarly. For a bifurcation configuration $\chi_{b}$ as shown in Fig.2(b), four selected points are the $p_{1}^{t h}, p_{2}^{t h}, p_{3}^{t h}$ and $p_{4}^{t h}$ points on $C_{k} . N_{k}$ edge points candidates on $C_{k}$ are separated into five parts by them. Sectional intensity profile of the two vessel branches are also estimated by the Gaussian intensity 
model in Eq.7. The likelihood function $P\left(Y_{k} \mid \chi_{b}\right)$ can be computed as:

$$
\begin{aligned}
P\left(Y_{k} \mid \chi_{b}\right) & =\prod_{i=1}^{p_{1}-1} P\left(y_{i} \mid b\right) \prod_{p_{1}}^{p_{2}} P\left(y_{i} \mid v\right) \prod_{p_{2}+1}^{p_{3}-1} P\left(y_{i} \mid b\right) \\
& \times \prod_{p_{3}}^{p_{4}} P\left(y_{i} \mid v\right) \prod_{p_{4}+1}^{N_{k}} P\left(y_{i} \mid b\right)
\end{aligned}
$$

For a crossing configuration, the likelihood function is obtained based on its six selected points.

\subsection{A Priori Probability}

The a priori probability of a configuration $\chi$ is expressed by Gibbs formulation [39]:

$$
P(\chi)=\frac{1}{Z} \exp (-\lambda U(\chi))
$$

where $Z$ is a normalization parameter and $\lambda$ is a weighting factor called regularization term. In this study, $Z$ and $\lambda$ were set to 1 and 0.01 respectively. $U(\chi)$ is the energy function of configuration $\chi$. The aim of using Gibbs formulation is to link a configuration with an energy function to penalize high energetic configurations.

In our study, the blood vessels are assumed to be locally linear, so local vessel edges can be estimated as two straight lines. At iteration $k(k \leq 5)$, vessel edge lines $L_{1}$ and $L_{2}$ are estimated as two straight lines along local direction $\overrightarrow{D_{k}}$ and passing through edge points $\hat{U}_{k}$ and $\hat{V}_{k}$ respectively. When $k>5, L_{1}$ and $L_{2}$ are defined as the least square straight lines obtained by five pairs of edge points detected in the previous iterations (see Fig.5). For a normal configuration $\chi_{n}$ with two selected points $M_{m_{1}}$ and $M_{m_{2}}$, we compute the distance between $M_{m_{1}}$ and $L_{1}$ and the distance between $M_{m_{2}}$ and $L_{2}$, which are denoted by $d_{m_{1}}$ and $d_{m_{2}}$ respectively. Energy $U\left(\chi_{n}\right)$ can be defined as: $U\left(\chi_{n}\right)=d_{m_{1}}^{2}+d_{m_{2}}^{2}$. So the configuration whose selected points aligned on the current edge lines is promoted. The a priori probability has the expression:

$$
P\left(\chi_{n}\right)=\frac{1}{Z} \exp \left(-\lambda\left(d_{m_{1}}^{2}+d_{m_{2}}^{2}\right)\right)
$$

For a bifurcation case, the estimated vessel edge lines are assumed to follow the outer edges of the bifurcation. Considering a bifurcation configuration 
$\chi_{b}$ with four selected points $M_{p_{1}}, M_{p_{2}}, M_{p_{3}}$ and $M_{p_{4}}$, the energy function is express as: $U\left(\chi_{b}\right)=d_{p_{1}}^{2}+d_{p_{4}}^{2}$, where $d_{p_{1}}$ is the distance between $L_{1}$ and $M_{p_{1}}$, and $d_{p_{4}}$ is the distance between $L_{2}$ and $M_{p_{4}}$. For a crossing case, the estimated edge lines are assumed to follow the edges of the main vessel. The other vessel which crosses the main one is independent to the estimated edge lines. Therefore, the energy function of the crossing configuration $\chi_{c}$ is express as: $U\left(\chi_{c}\right)=d_{q_{3}}^{2}+d_{q_{4}}^{2}$. Then the a priori probability for the bifurcation or crossing configuration can be calculated by Eq.12.

\section{Experiments and discussion}

The performance of our method was evaluated on both simulated and real retinal images. In our study, we obtained a simulated image from a noise-free simulated vascular image which represents different vessel structures [40]. The real retinal images were downloaded from REVIEW Database (http://reviewdb.lincoln.ac.uk), a public database focusing on the development and comparison of vessel measurement algorithms.

\subsection{Experiments on simulated image}

The simulated vascular image is shown in Fig.6(a). It has seven segments with different geometric shapes to model different vessel features in retinal images. Four segments on the first row describe a single linear vessel, a vessel bifurcation with two branches, a crossing structure and a linear vessel with varying diameters respectively. The other three segments on the second row simulate blood vessels with different curvatures. The sectional intensity distribution of these segments follows Gaussian distribution. In practice, we added Gaussian white noise on the simulated vascular image for the test purpose.

In order to characterize the algorithm performance, we used the Segmentation Matching Factor (SMF) as a quality parameter [33].

$$
S M F=\frac{\operatorname{Card}\left(\Phi_{\text {sim }} \bigcap \Phi_{\text {seg }}\right)}{\operatorname{Card}\left(\Phi_{\text {sim }} \bigcup \Phi_{\text {seg }}\right)}
$$

where $\Phi_{\text {sim }}$ and $\Phi_{\text {seg }}$ are the sets of pixels belonging to the simulated and the segmented blood vessels respectively. $\operatorname{Card}(\Phi)$ is the number of elements in set $\Phi$. SMF equals 1 in the case of a perfect segmentation, 0 if segmentation fails. 
For example, we applied our method on a noisy image with $\mathrm{SNR}=5 d B$ as shown in Fig.6(b). Initial points for all the seven segments are first selected manually. Then edge points of the seven segments are detected respectively by the proposed tracking method. From Fig.6(c), we can see that our method detected all the vessel segments with different vessel structures and varying diameters and curvatures. In this study, we join edge detected points and fill regions inside these obtained edge lines to get the segmented blood vessels. Fig.6(d) gives the segmented result of the proposed method and SMF value is calculated by Eq.14.

Next we tested the influence of noise upon quality parameter SMF. For different given Gaussian noise (SNR from $1 d B$ to $12 d B$ ), SMF values were computed. If we assume a constant intensity model (sectional intensity inside the vessel and in the background are two different constant values) [41] instead of the Gaussian intensity model used for this study, less efficient results are obtained. Fig.7 shows the results on simulated images, using the proposed method, proposed method with constant intensity model, Sun's method [42] and Chaudhuri's method [2]. Sun in [42] proposed a trackingbased method, which estimates vessel boundaries by analyzing the pixels belonging to a scanline orthogonal to the tracking direction. Chaudhuri's method is a filter-based method, which uses the 2-D Gaussian filter to match vessels for detection.

As it can be noticed from the result curves, efficiency of the proposed method increases when SNR is higher and the SMF value can reach 0.85 when SNR is higher than $5 d B$. The proposed method gave obviously better results than the others. When SNR was lower than 5, the SMF values of the proposed method were all higher than 0.7, while the SMF values of other methods decreased and were all lower than 0.7. Besides, the efficiency of the proposed method with constant intensity model was better than Sun's [42] and Chaudhuri's [2] methods. From the comparison, we can conclude that the proposed method increases the detection accuracy and the application of Gaussian intensity model improves the robustness against noise.

\subsection{Experiments on retinal image}

On the other hand, we applied our algorithm on retinal images obtained from REVIEW database. REVIEW is a retinal vessel reference database. It consists of four image sets (HRIS, VDIS, CLRIS, KPIS) which include 16 images with 193 vessel segments demonstrating a variety of pathologies and vessel types. These image sets contain 5066 manually marked profiles 
assessed by three independent experts. The performance of an algorithm on the retinal image can be compared with manual measurement with accuracy up to 0.25 of a pixel.

In practice, we used the green channel of the color fundus images, because this channel has the highest contrast between regions belonging to the blood vessel and the background [3]. Fig.8 shows some test results on a retinal image from REVIEW database. We can see that different vessel structures and vessel edge points are well detected by the proposed tracking algorithm.

Next we used the high resolution image set (HRIS). It includes four images where 90 segments containing 2368 pairs of edge points are manually marked. The 90 segments are all isolated linear vessel segments which are selected manually by the experts of REVIEW database. The purpose of using these vessel segments and corresponded ground truth is to evaluate the detection accuracy. The edge points of three observers provide the information of vessel center point, local width and local direction. When testing our method on the retinal images in HRIS, the first pair of manually marked edge points of each segment is used for the initialization. Because the length of each segment is fixed, for comparison purpose, the tracking process is stopped when the detected edge points exceed the vessel segment. Fig.9 gives an example of the tracking process of a vessel segment in HRIS001, which is the first image in HRIS. In this figure, we can see from Fig.9(a) that the interested vessel segment starts from a point in the black circle. Initial data of the tracking process as shown in Fig.9(b) are obtained by vessel segment's first pair of marked edge points. In Fig.9(c), a semi-ellipse and two edge lines are drawn. New edge points are selected from the edge points candidates on this semi-ellipse by the proposed tracking method. Fig.9(d) shows the edge points detected by the proposed method and true vessel edge lines marked by the observers. The test result in Fig.9 is obtained by stopping the proposed tracking method manually. If the tracking algorithm starts from an initial point on the same image HRIS001 without ending manually, local vascular tree can be obtained as shown in Fig.10. The image shown in Fig.10(a) is a subimage extracted from Fig.9(a), and the initial point is marked by a black circle. Segmentation results of the proposed method and Adel's method [33] are shown in Fig.10(b) and (c), respectively. It's obvious that our method detects different vessel structures and has a higher accuracy in vessel detection than Adel's method [33].

For evaluation purpose, we performed a ROC (Receiver Operating Characteristic) analysis to assess the accuracy of our method. If a pixel in detected 
blood vessel belongs to true blood vessel, it is called true positive pixel. Otherwise, it is considered as false positive pixel if it does not belong to true blood vessel. True positive rate (TPR) is defined as the ratio between the number of true positive pixels and the total number of pixels in true blood vessel. False positive rate (FPR) is the ratio between the number of false positive pixels and the total number of pixels in the true background. We tested our method on the 90 vessel segments on four retinal images in HRIS and computed the corresponding TPR and FPR values. As mentioned in the previous section, Sun's method [42] is a well-known tracking-based method, which is often chosen as a compared method when testing on the real medical images [37, 43]. We also applied Sun's method on HRIS for comparison purposes. Some examples of vessel segments in HRIS are illustrated in Fig.11. Subfigures on the first row show the edge points marked by observers on different vessel segments including linear vessel and vessels with curvature, with low contrast between vessel and background and with small diameters respectively. Subfigures on the second and third rows show detected results using the proposed method and Sun's method respectively. Our method is more precise than Sun's. Even Sun's method failed, our method still can work well (see Fig.11(g) and $(\mathrm{k})$ ). During the tracking process of the proposed method, both the grey level statistics in the local search area and the information from the previous iterations are used. However, only the pixels belonging to the scanlines are considered in Sun's tracking method, which makes Sun's method more sensitive to the noise. Table.1 shows the mean TPR and FPR values obtained using the proposed method and Sun's method on the four retinal images in HRIS. The ROC figures of two methods are shown in Fig.12. We plotted the test results on 90 vessel segments in HRIS as points in ROC space. If a method yield a point nearer to the upper left corner of the ROC space, it is better than others. So, from the results shown in Fig.12, our method performed better than Sun's method.

\section{Conclusion}

In this paper, we proposed a probabilistic tracking method for retinal vessel segmentation. During the tracking process, a semi-ellipse was used as the dynamic search window to get the information of local grey levels statistics. We defined a concept of configuration based on the points candidates on a semi-ellipse. Different types of configurations combined the intensity distribution and the geometrical structure of local blood vessel. Vessel's edge 
points were detected by a Bayesian method with the MAP criterion. The proposed method detected the blood vessels effectively on both simulated and real retinal images, and it performed better than some classical vessel detection methods. In the future, we will consider the relationship between the local grey levels statistics to improve the performance instead of considering the assumption of grey levels profile independent used in this work (Eq.5). Local vessel edges are estimated as two least square lines. This model is simple and did not involve the information of local vessel's curvature. The improvement of the a priori model is also a necessary following work.

\section{References}

[1] C. Kirbas, F. Quek, A review of vessel extraction techniques and algorithms, ACM Comput. Surv. 36 (2004) 81-121.

[2] S. Chaudhuri, S. Chatterjee, N. Katz, M. Nelson, M. Goldbaum, Detection of blood vessels in retinal images using two-dimensional matched filters, IEEE Trans. Med. Imaging 8 (1989) 263-269.

[3] A. Hoover, V. Kouznetsova, M. Goldbaum, Locating blood vessels in retinal images by piecewise threshold probing of a matched filter response, IEEE Trans. Med. Imaging 19 (2000) 203-210.

[4] F. Miles, A. Nutall, Matched filter estimation of serial blood vessel diameters from video images, IEEE Trans. Med. Imaging 12 (1993) $147-152$.

[5] L. Zhou, M. Rzeszotarski, L. Singerman, J. Chokreff, The detection and quantification of retinopathy using digital angiograms, IEEE Trans. Med. Imaging 13 (1994) 619-626.

[6] X. Gao, A. Bharath, A. Hughes, A. Stanton, N. Chapman, S. Thom, Towards retinal vessel parametrisation, SPIE Conference on Medical Imaging (1997) 734-744.

[7] X. Gao, A. Bharath, A. Stanton, A. Hughes, N. Chapman, S. Thom, Quantification and characterisation of arteries in retinal images, Comput Methods Programs Biomed. 63 (2000) 133-146.

[8] Y. Tolias, S. Panas, A fuzzy vessel tracking algorithm for retinal images based on fuzzy clustering, IEEE Trans. Med. Imaging 17 (1998) 263-273. 
[9] X. Jiang, D. Mojon, Adaptive local thresholding by verificationbased multithreshold probing with application to vessel detection in retinal images, IEEE Trans. Pattern Anal. Mach. Intell. 25 (2003) 131-137.

[10] A. Can, H. Shen, J. N. Turner, H. L. Tanenbaum, B. Roysam, Rapid automated tracing and feature extraction from live high-resolution retinal fundus images using direct exploratory algorithms, IEEE T. Inf. Technol. Biomed. 3 (1999) 125-138.

[11] D. Koozekanani, K. Boyer, C. Roberts, Retinal thickness measurements from optical coherence tomography using a markov boundary model, IEEE Trans. Med. Imaging 20 (2001) 900-916.

[12] M. E. Martínez-Pérez, A. D. Hughes, A. V. Stanton, S. A. Thom, A. A. Bharath, K. H. Parker, Retinal blood vessel segmentation by means of scale-space analysis and region growing, In Proceedings of the 2nd International Conference of Medical Image Computing and ComputerAssisted Intervention (MICCAI 1999) (1999) 90-97.

[13] V. Mahadevan, H. Narasimha-Iyer, B. Roysam, H. Tanenbaum, Robust model-based vasculature detection in noisy biomedical images, IEEE T. Inf. Technol. Biomed. 8 (2004) 360-376.

[14] C. Heneghan, J. Flynn, M. O'Keefe, M. Cahill, Characterization of changes in blood vessel width and tortuosity in retinopathy of prematurity using image analysis, IEEE T. Inf. Technol. Biomed. 6 (2002) 407-429.

[15] F. Zana, J. Klein, Robust segmentation of vessels from retinal angiography, Proceedings of the International Conference on Digital Signal Processing (1997) 1087-1090.

[16] S. Aylward, E. Bullitt, Initialization, noise, singularities, and scale in height-ridge traversal for tubular object centerline extraction, IEEE Trans. Med. Imaging 21 (2002) 61-75.

[17] J. Jomier, D. K. Wallace, S. R. Aylward, Quantification of retinopathy of prematurity via vessel segmentation, In Proceedings of the 6th International Conference of Medical Image Computing and ComputerAssisted Intervention (MICCAI 2003) (2003) 620-626. 
[18] J. Staal, M. Abramoff, M. Niemeijer, M. Viergever, B. van Ginneken, Ridge-based vessel segmentation in color images of the retina, IEEE Trans. Med. Imaging 23 (2004) 501-509.

[19] J. Staal, S. Kalitzin, M. A. Viergever, A trained spin-glass model for grouping of image primitives, IEEE Trans. Pattern Anal. Mach. Intell. 27 (2005) 1172-1182.

[20] J. V. B. Soares, J. J. G. Leandro, R. M. C. Jnior, H. F. Jelinek, M. J. Cree, Retinal vessel segmentation using the 2-d gabor wavelet and supervised classification, IEEE Trans. Med. Imaging 25 (2006) 1214-1222.

[21] E. Ricci, R. Perfetti, Retinal blood vessel segmentation using line operators and support vector classification, IEEE Trans. Med. Imaging 26 (2007) 1357-1365.

[22] A. Mendonca, A. Mendonca, A. Campilho, Segmentation of retinal blood vessels by combining the detection of centerlines and morphological reconstruction, IEEE Trans. Med. Imaging 25 (2006) 1200-1213.

[23] M. E. Martínez-Pérez, A. D. Hughes, S. A. Thom, A. A. Bharath, K. H. Parker, Segmentation of blood vessels from red-free and fluorescein retinal images, Med. Image Anal. 11 (2007) 47-61.

[24] A. B. L. Wang, R. Wilson, Analysis of retinal vasculature using a multiresolution hermite model, IEEE Trans. Med. Imaging 26 (2007) 137152.

[25] U. K. D. Adjeroh, J. Odom, Texton-based segmentation of retinal vessels, J. Opt. Soc. Am. A 24 (2007) 1384-1393.

[26] M. Niemeijer, B. van Ginneken, M. Abràmoff, A linking framework for pixel classification based retinal vessel segmentation, SPIE Medical Imaging 7262 (2009) 726216.

[27] O. Chutatape, L. Zheng, S. Krishnan, Retinal blood vessel detection and tracking by matched gaussian and kalman filters, Proceedings of the 20th Annual International Conference of the IEEE EMBS 20 (1998) 3144-3149. 
[28] E. Grisan, A. Pesce, A. Giani, M. Foracchia, A. Ruggeri, A new tracking system for the robust extraction of retinal vessel structure, Proceedings of the 26th Annual International Conference of the IEEE EMBS 3 (2004) $1620-1623$.

[29] J. Ng, S. Clay, S. Barman, A. Fielder, M. Moseley, K. Parker, C. Paterson, Maximum likelihood estimation of vessel parameters from scale space analysis, image and vision computing, Image Vis. Comput. 28 (2010) 56-63.

[30] H. Li, W. Hsu, M. L. Lee, H. Wang, A piecewise gaussian model for profiling and differentiating retinal vessels, International Conference on Image Processing (2003) 1069-1072.

[31] A. D. Flemming, S. Philip, K. A. Goatman, J. A. Olson, P. F. Sharp, Automated microaneurysm detection using local contrast normalization and local vessel detection, IEEE Trans. Med. Imaging 25 (2006) 12231232 .

[32] S. Zhou, W. Chen, Z. Zhang, J. Yang, Segmentation of coronary angiograms based on probabilistic tracking, International Conference on Automatic Bioinformatics and Biomedical Engineering 25 (2009) 1-4.

[33] M. Adel, A. Moussaoui, M. Rasigni, S. Bourennane, L. Hamami, Statistical-based tracking technique for linear structures detection: Application to vessel segmentation in medical images, IEEE Signal Process. Lett. 17 (2010) 555-558.

[34] R. Pajak, Use of two-dimensional matched filters for estimating a length of blood vessels newly created in angiogenesis process, Opto-Electronics Review 11 (2003) 237-241.

[35] Y. Bouaoune, M. K. Assogba, J. C. Nunes, P. Bunel, Spatio-temporal characterization of vessel segments applied to retinal angiographic images, Pattern Recogn. Lett. 24 (2003) 607-615.

[36] K. A. Vermeer, F. M. Vos, H. G. Lemij, A. M. Vossepoel, A model based method for retinal blood vessel detection, Computers in Biology and Medicine 34 (2004) $209-219$. 
[37] P. Zou, P. Chan, P. Rockett, A model-based consecutive scanline tracking method for extracting vascular networks from 2-d digital subtraction angiograms, IEEE Transactions on Medical Imaging 28 (2009) 241-249.

[38] J. Lowell, A. Hunter, D. Steel, A. Basu, R. Ryder, R. Kennedy, Measurement of retinal vessel widths from fundus images based on 2-d modeling, IEEE Trans. Med. Imaging 23 (2004) 1196-1204.

[39] M. Ibanez, A. Simo, Bayesian detection of the fovea in eye fundus angiographies, Pattern Recognit. Lett. 20 (1999) 229-240.

[40] P. T. Truc, M. A. Khan, Y.-K. Lee, S. Lee, T.-S. Kim, Vessel enhancement filter using directional filter bank, Comput. Vis. Image Underst. 113 (2009) 101-112.

[41] Y. Yin, M. Adel, M. Guillaume, S. Bourennane, A probabilistic based method for tracking vessels in retinal images, IEEE International Conference on Image Processing (2010) 4081-4084.

[42] Y. SUN, Automated identification of vessel contours in coronary arteriograms by an adaptive tracking algorithm, IEEE Trans. Med. Imaging 8 (1989) 78-88.

[43] Y. Xu, H. Zhang, H. Li, G. Hu, An improved algorithm for vessel centerline tracking in coronary angiograms, Computer Methods and Programs in Biomedicine 88 (2007) 131-143. 


\section{Figure Captions}

Fig.1: Statistical sampling scheme: $\hat{U}$ and $\hat{V}$ are the vessel edge points, $O$ is the center point, $\vec{D}$ is the vessel direction, and $k$ is the index of the iteration. $C_{k}$ is the semi-ellipse at iteration $k . a_{k}$ and $b_{k}$ are the major and minor axes of $C_{k}$, respectively. Black points on $C_{k}$ show the possible locations of new edge points.

Fig.2: Three types of configurations: (a) normal; (b) bifurcation; (c) crossing. $O_{k}$ is local center point, $C_{k}$ is the dynamic search window, points marked by small circles are used to define different types of configurations.

Fig.3: Sectional intensity profile of a blood vessel: (a) Gaussian intensity model; (b) the real and estimated intensity profile of the selected vessel cross-section.

Fig.4: Estimation of the statistic parameters on three regions.

Fig.5: Least square lines obtained by the previous vessel edge points. $M_{m_{1}}$ and $M_{m_{2}}$ are a pair of selected candidate edge points, which are used to define a normal configuration $\chi_{n}$.

Fig.6: Test on simulated images: (a) simulated vascular image with seven types of segments; (b) noisy image with $S N R=5 d B$; (c) edge points detected by the proposed method; (d) segmented result with $S M F=0.852$.

Fig.7: SMF at different SNR values using different vessel detection method.

Fig.8: Detection of different vessel structures on real retinal image: (a) and (b) single vessel; (c) bifurcation; (d) crossing. Semi-ellipse search windows are shown for different vessel structures and the edge points detected on the semi-ellipse are marked by small circles.

Fig.9: Tracking of a vessel segment: (a) retinal image HRIS001; (b) initialization of the proposed method; (c) dynamic search window and estimated edge lines at a given iteration; (d) edge points detected by the proposed 
method and the edge lines obtained by the observers.

Fig.10: Local vessel segmentation: (a) local retinal image; (b), (c) segmentation results of the proposed method and Adel's method [33], respectively.

Fig.11: Examples of vessel segments in REVIEW database: (a),(b),(c),(d) edge points marked by the observer; (e),(f),(g),(h) edge points detected by the proposed method; (i),(j),(k),(l) edge points detected by Sun's method.

Fig.12: ROC figures of the experiments on REVIEW database: (a) proposed method; (b) Sun's method. 


\section{Table Captions}

Table.1: Evaluation results on four retinal images in HRIS. 


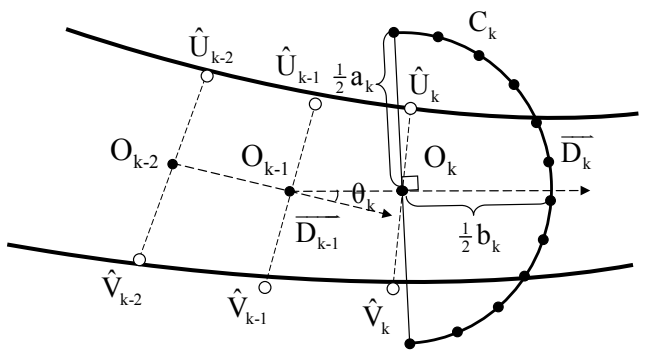

Figure 1: 


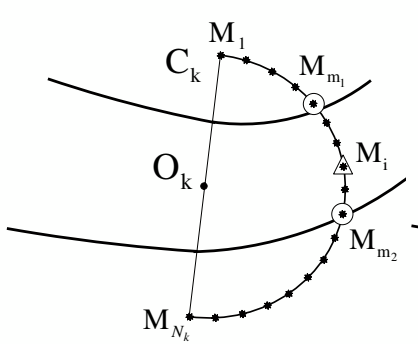

(a)

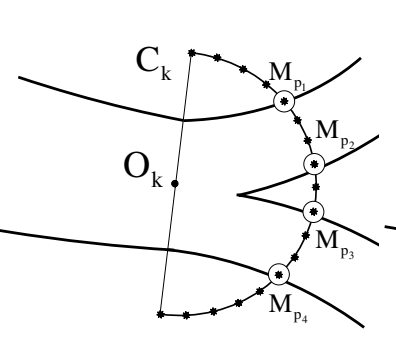

(b)

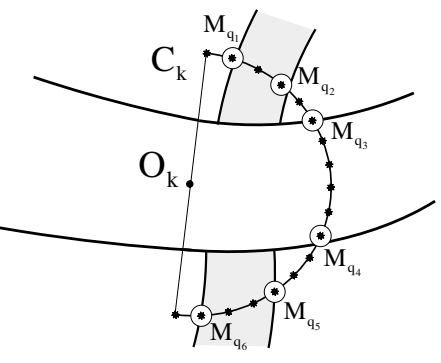

(c)

Figure 2: 


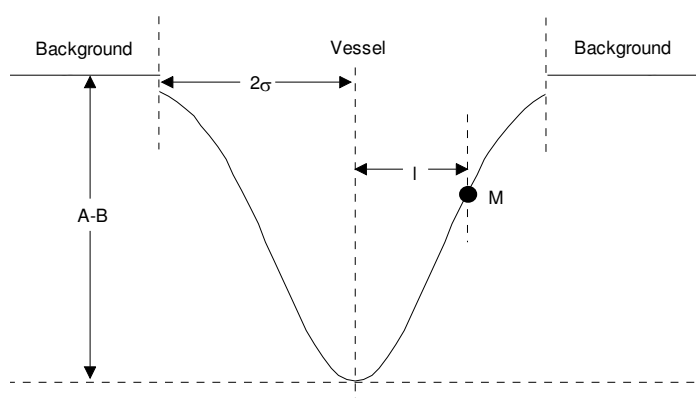

(a)
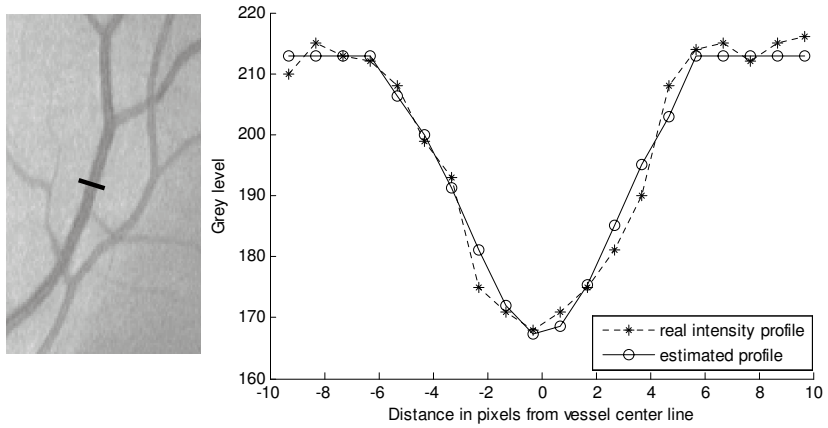

(b)

Figure 3: 


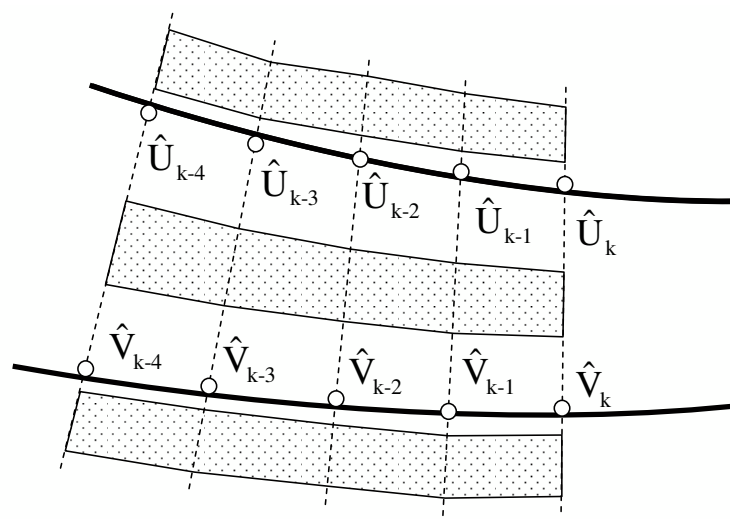

Figure 4: 


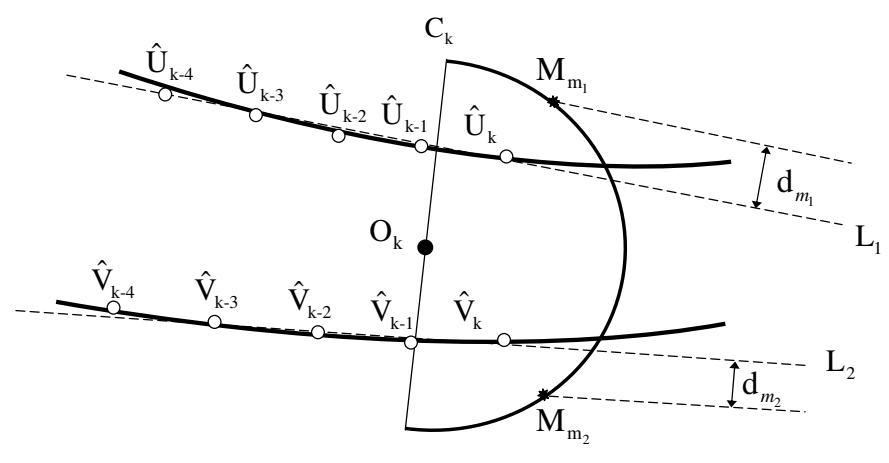

Figure 5: 

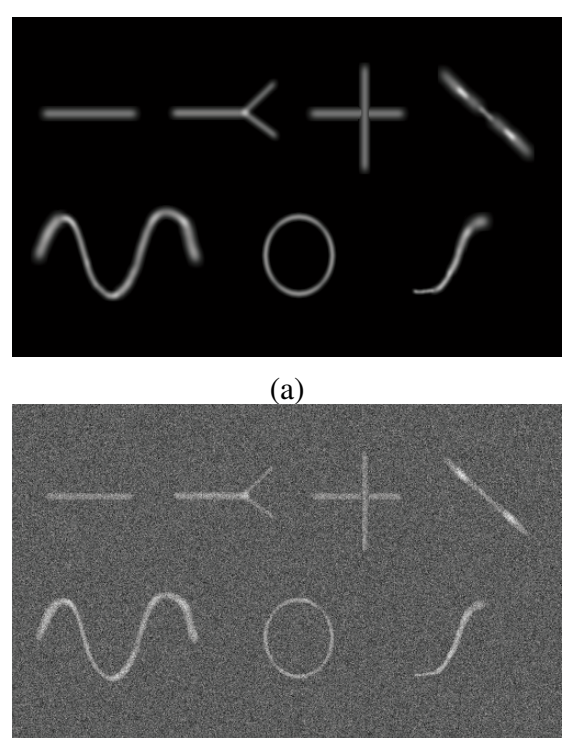

(b)

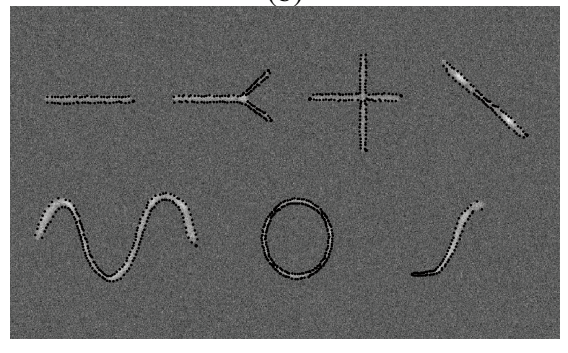

(c)

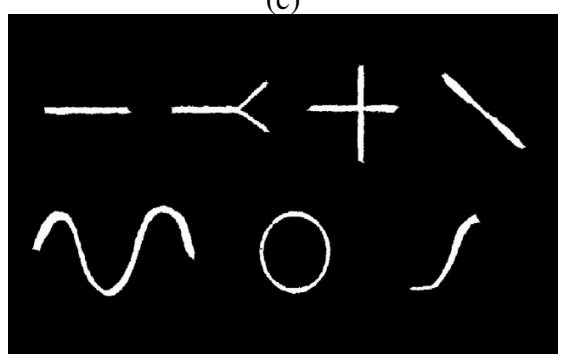

(d)

Figure 6: 


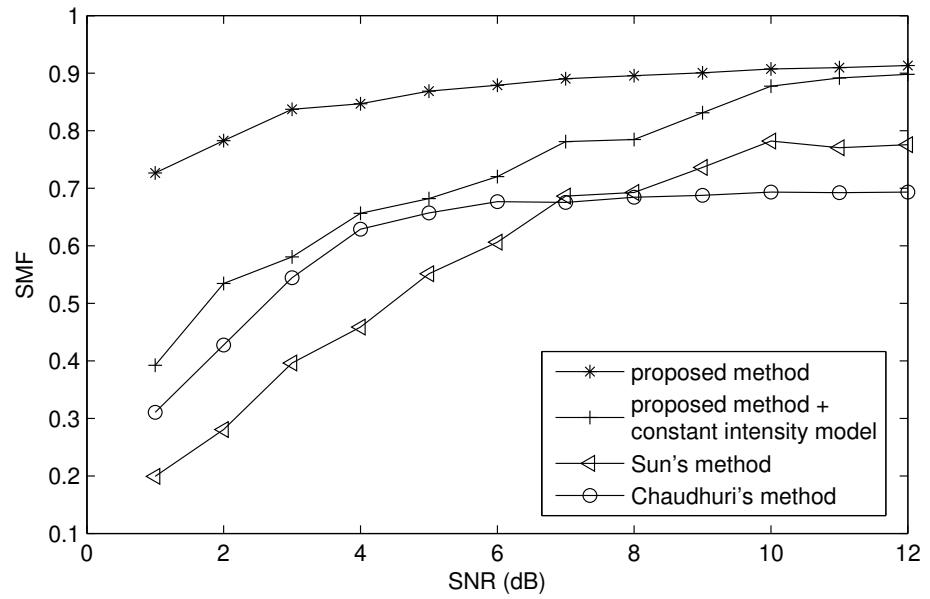

Figure 7: 


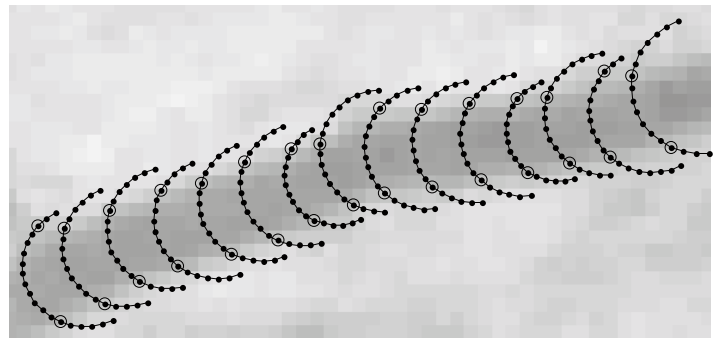

(a)

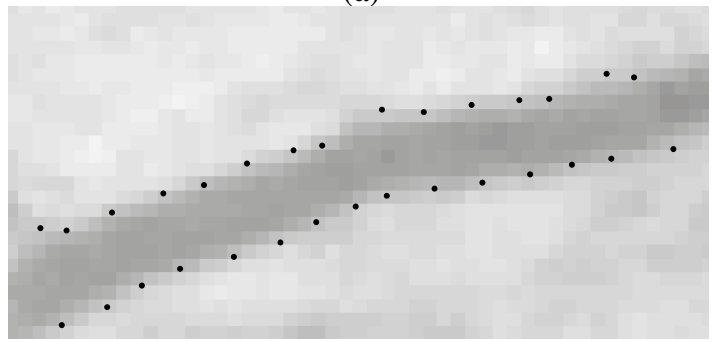

(b)

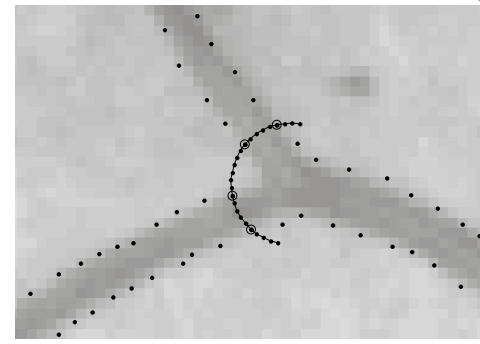

(c)

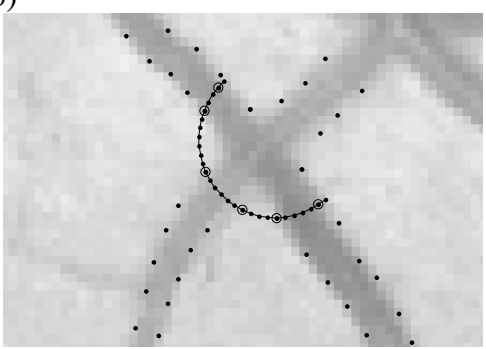

(d)

Figure 8: 


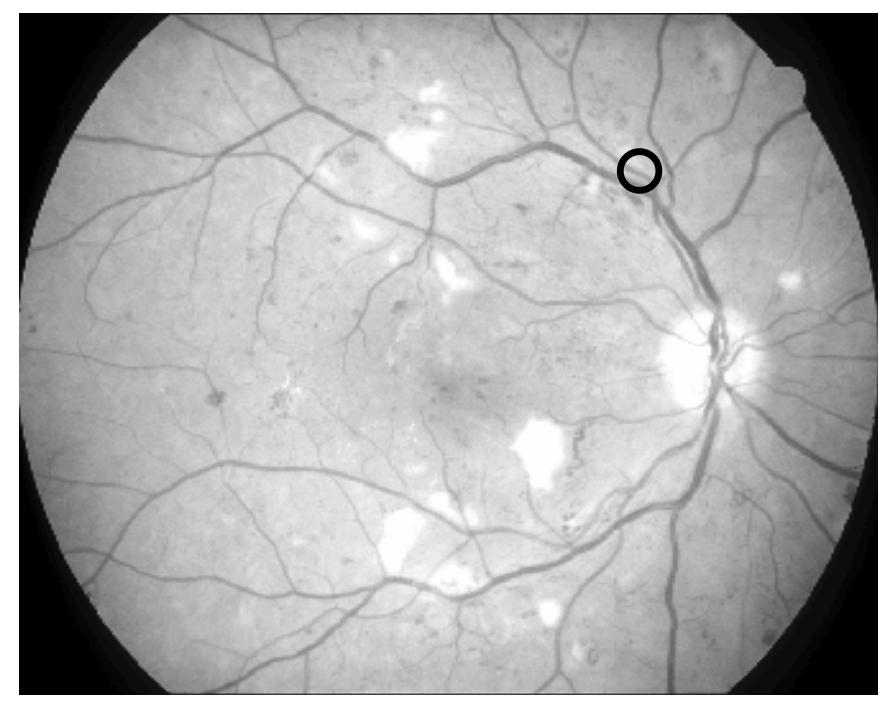

(a)

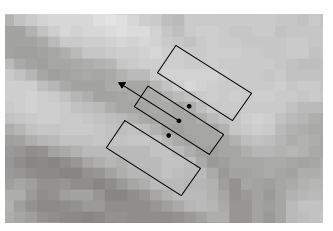

(b)

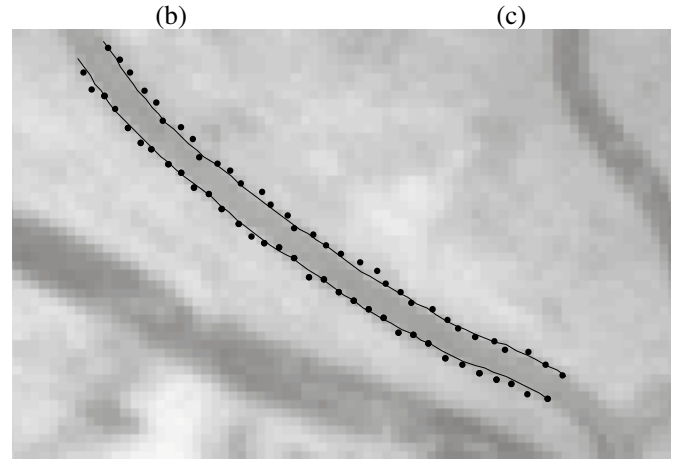

(d)

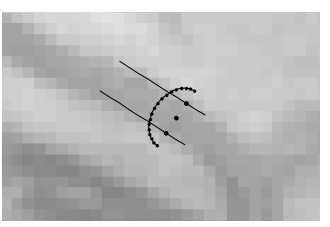

(c)

Figure 9: 


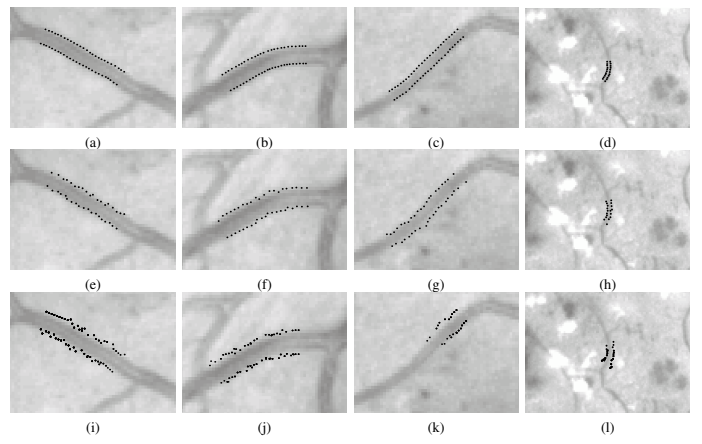

Figure 10: 


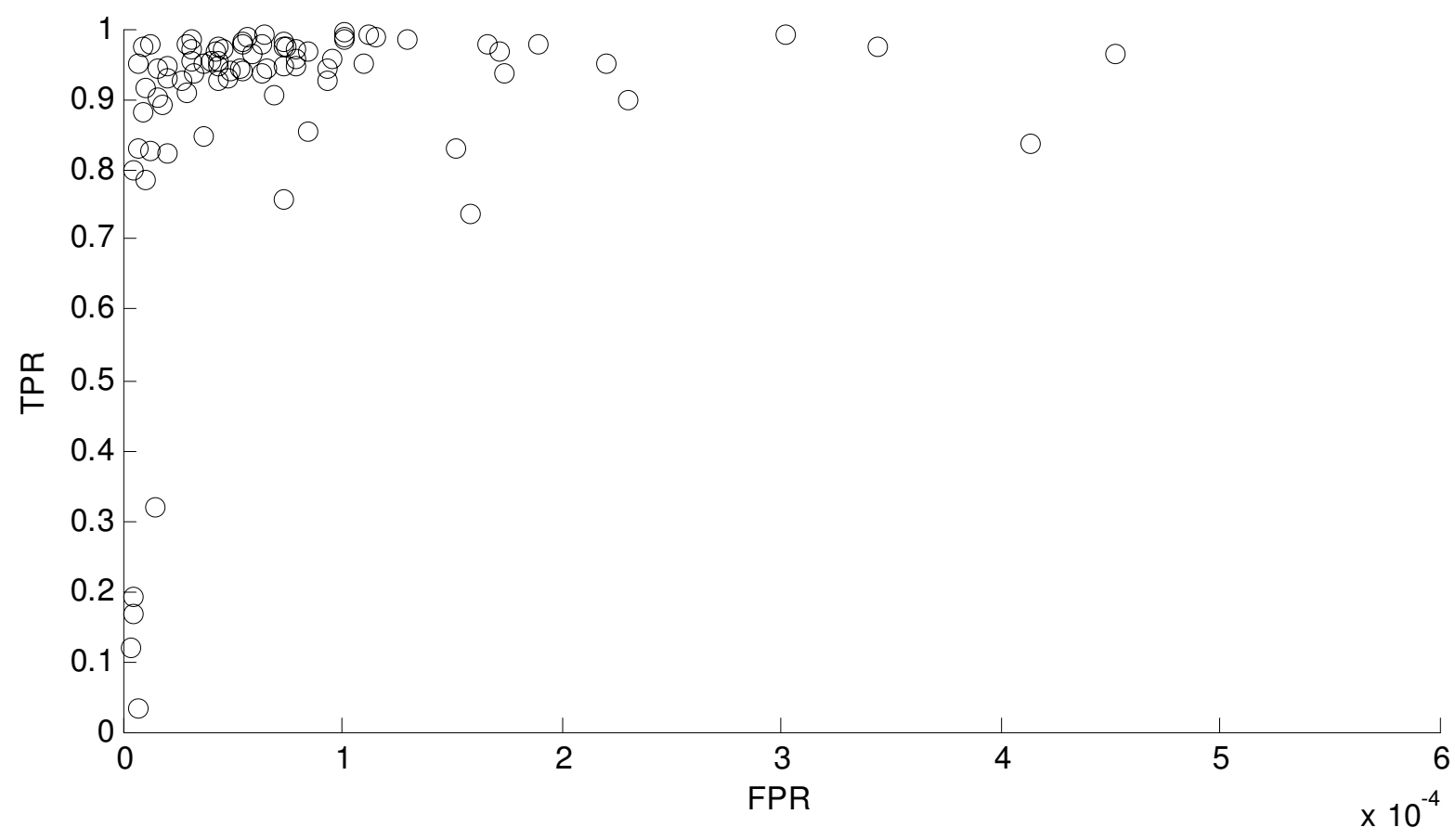

(a)

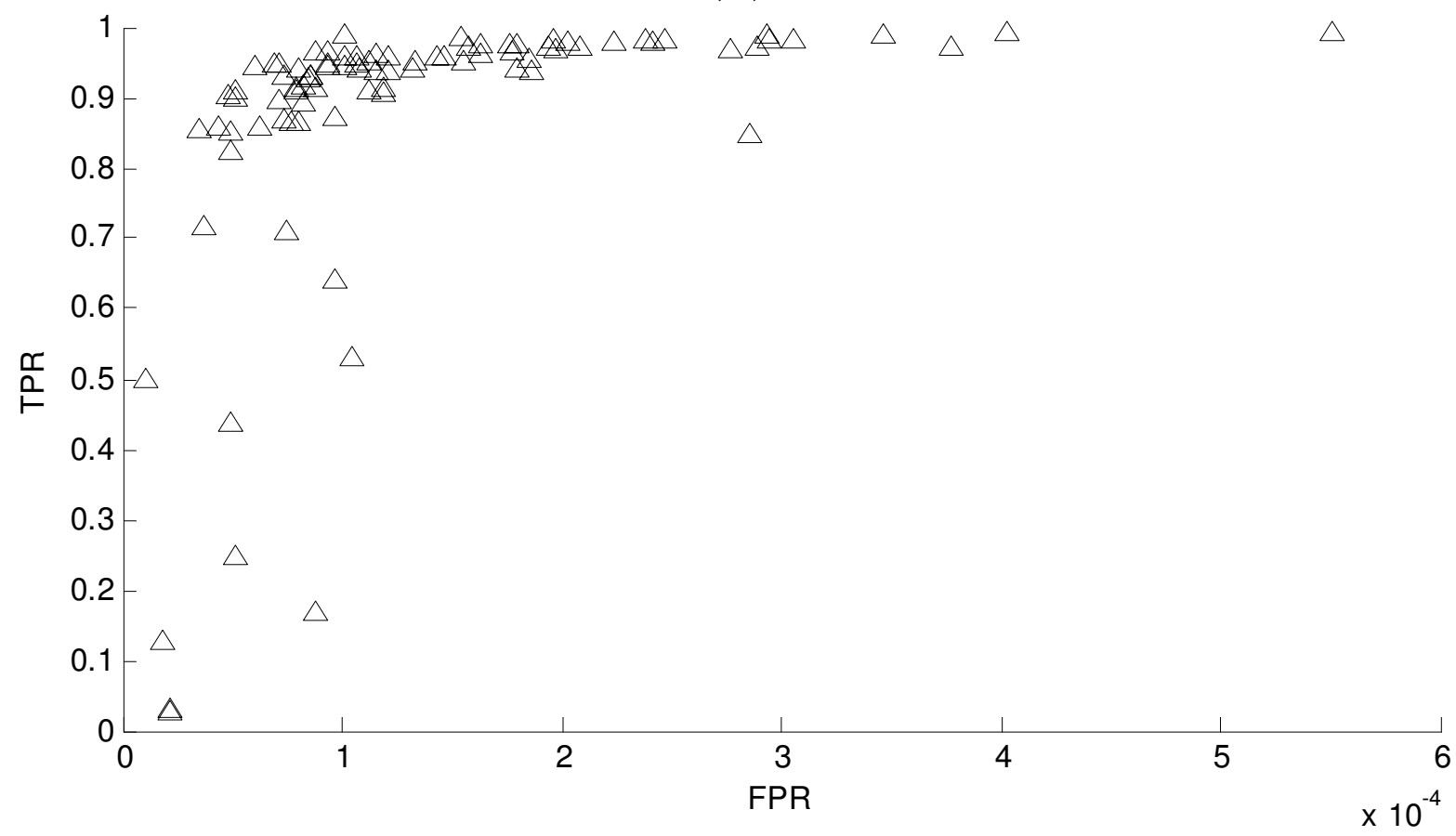

(b)

Figure 11: 


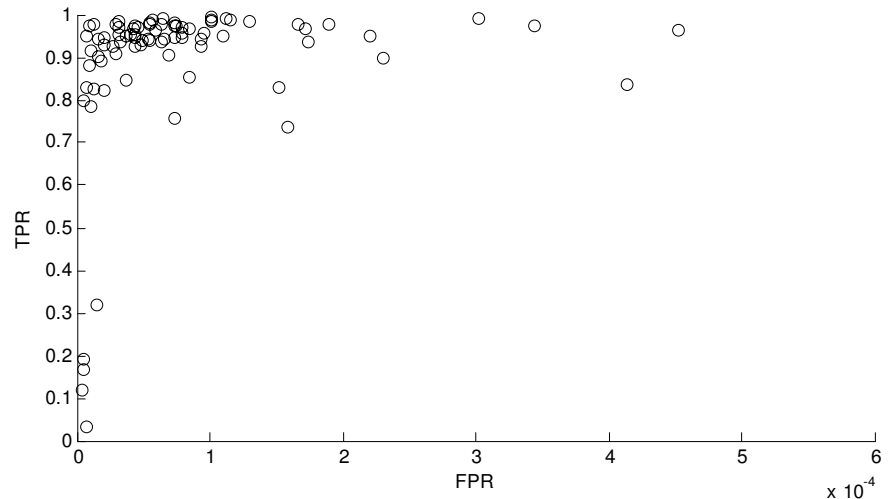

(a)

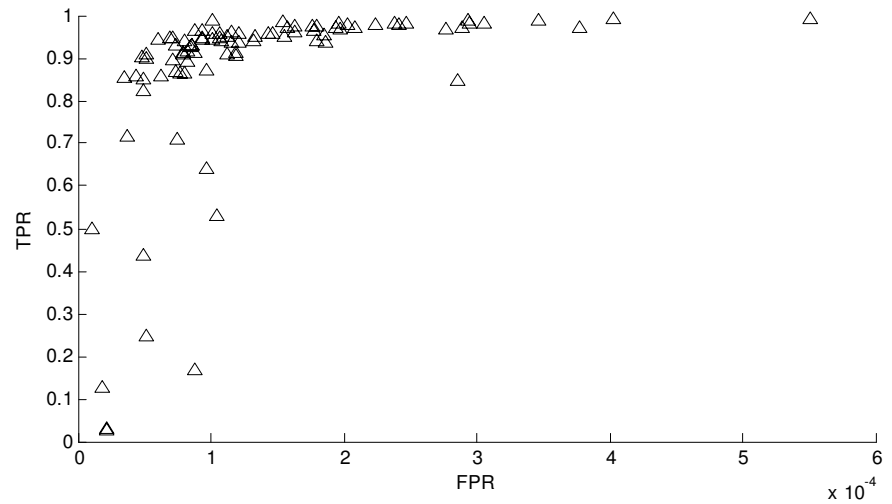

(b)

Figure 12: 


\begin{tabular}{|c|c|c|c|c|c|c|c|c|c|c|}
\hline & \multicolumn{2}{|c|}{ HRIS001 } & \multicolumn{2}{c|}{ HRIS002 } & \multicolumn{2}{c|}{ HRIS003 } & \multicolumn{2}{c|}{ HRIS004 } & \multicolumn{2}{c|}{ Mean } \\
\cline { 2 - 11 } & TPR & FPR & TPR & FPR & TPR & FPR & TPR & FPR & TPR & FPR \\
\hline Proposed Method & 0.909 & $8.11 \times 10^{-5}$ & 0.943 & $4.76 \times 10^{-5}$ & 0.887 & $8.11 \times 10^{-5}$ & 0.96 & $3.89 \times 10^{-5}$ & 0.925 & $6.21 \times 10^{-5}$ \\
\hline Sun's Method & 0.842 & $1.45 \times 10^{-4}$ & 0.905 & $1.16 \times 10^{-4}$ & 0.871 & $1.36 \times 10^{-4}$ & 0.966 & $9.56 \times 10^{-5}$ & 0.895 & $1.23 \times 10^{-4}$ \\
\hline
\end{tabular}

Table 1: 